\title{
Clinical Application of the 2011 Cancer Cachexia Consensus
}

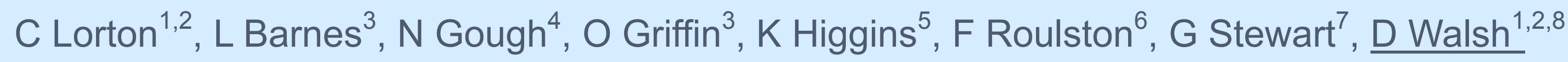

1. Our Lady's Hospice \& Care Services, Dublin; 2. School of Medicine, Trinity College Dublin; 3. St Vincent's University Hospital, Dublin; 4. Mater Private Mid-Western Radiation Oncology Centre, Limerick; 5. Tallaght Hospital, Dublin; 6. St Luke's Radiation Oncology Network, Dublin; 7. St Vincent's Private Hospital, Dublin; 8. School of Medicine and Medical Science, University College Dublin

\section{BACKGROUND}

Cancer cachexia is prevalent

- Poor prognosis \& $\downarrow$ quality of life

2011 consensus definition and classification ${ }^{1}$

- Defined stages and assessment domains

- Objective assessment muscle mass

- not usual in clinical practice

- Unknown if assessment domains and staging feasible in routine clinical practice

\section{METHODS}

- 5 tertiary referral centres

- Consecutive referrals to Oncology dietitian

- Adults with solid tumours

- Data collected during routine first assessment

\section{OBJECTIVE}

Assess if Oncology dietitians could classify cachexia stage, as part of routine first assessment, based on 2011 consensus ${ }^{1}$

Classify as

- No cachexia

- Pre-cachexia

- Cachexia

- Refractory cachexia

- OR unable to classify and why not

Dietitian classified cachexia stage based on

- weight, weight loss

- food intake, nutritional intake symptoms

- systemic inflammation

- functional ability

- disease status

\section{RESULTS}

$\mathrm{n}=200$ (female 119, male 81)

Median age 65 (Range: 19-93)

$49 \%$ metastatic disease, $91 \%$ active treatment $40 \%$ overweight or obese

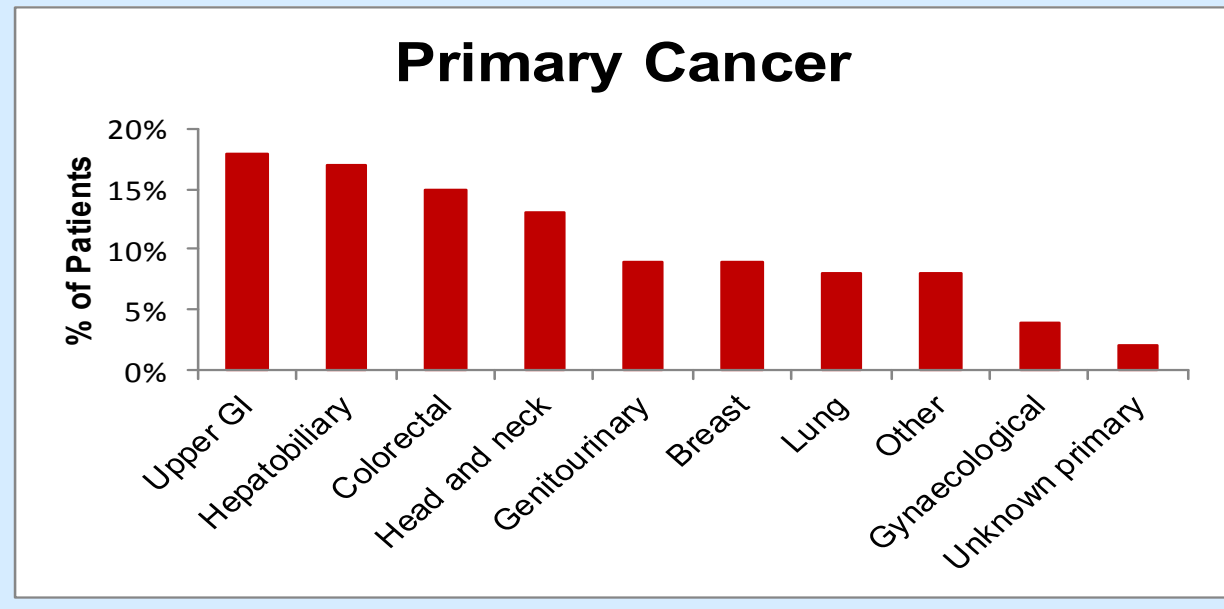

\section{Cachexia Classification}

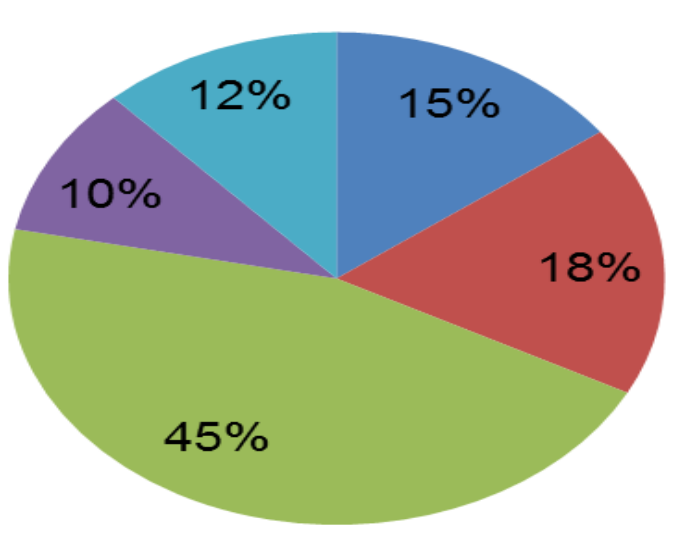

- No cachexia

- Pre-cachexia

- Cachexia

- Refractory

cachexia

- Unable to classify

\begin{tabular}{|l|l|}
\hline Reason unable to classify & Number of times cited \\
\hline No CRP & 14 \\
\hline No albumin & 2 \\
\hline Missing weight loss info & 2 \\
\hline Complex clinical picture & 4 \\
\hline Acutely ill & 2 \\
\hline
\end{tabular}

\section{CONCLUSIONS}

- 2011 cancer cachexia consensus classification was surprisingly usable in clinical setting

- Oncology dietitians were able to use routine clinical information to classify $88 \%$ of patients

- Almost two-thirds were considered cachectic

- Future studies should validate clinical assessment against outcomes and objective muscle mass

- Expert clinical assessment based on 2011 consensus could be cost-effective to screen for cachexia

Acknowledgements: Martina Coen, Clare Corish, Catherine Corrigan, Sinéad Feehan, Una Gilligan, Geraldine Guiry, Orla Haughey, Shauna 